\title{
Exploiting the Power of Sensory-Motor Coordination
}

\author{
Stefano Nolfi ${ }^{1}$, Domenico Parisi ${ }^{1}$ \\ ${ }^{1}$ Institute of Psychology, National Research Council (CNR), \\ 15 Viale Marx, 00137, Rome, Italy \\ \{nolfi, parisi\}@ip.rm.cnr.it
}

\begin{abstract}
One important implication of embodiment is that, by acting, agents partially determine the sensory patterns they receive from the environment. The motor actions performed by an agent, by modifying the agent's position with respect to the external environment and/or the external environment itself, partially determine the type of sensory patterns received from the environment. In this paper we investigate how agents can take advantage of this ability. In particular, we discuss how agents coordinate sensory and motor processes in order to (1) select sensory patterns which are not affected by the aliasing problem and avoid those which are; (2) select sensory patterns such that groups of patterns which require different responses do not strongly overlap; (3) exploit emergent behaviors that result from the interaction between the agent and the environment.
\end{abstract}

\section{Introduction}

Recently, a new research paradigm has challenged the traditional view according to which intelligence is an abstract process that can be studied without taking into consideration the physical aspects of natural systems. In this new paradigm, research tends to stress the importance of situatedness (i.e. the importance of studying systems, natural or artificial, which are situated within an external environment) and embodiment (i.e. the importance of study systems which have bodies, receive input from their sensors and produce motor actions as outputs). We will refer to systems which are embodied and situated as agents.

One important implication of embodiment is that, by acting, agents partially determine the sensory patterns they receive from the environment. The motor actions performed by an agent, by modifying its position with respect to the external environment and/or the external environment itself, in fact partially determine the type of sensory patterns that it will receive from the environment. As we will see below, agents can take advantage of this ability in different ways. We will refer to the process of exploiting the agent-environment interaction (i.e. the ability to select sensory patterns that are useful for some purpose through certain motor actions) as sensory-motor coordination (for a similar view see [1]). We will show three different ways in which sensory-motor coordination can help to solve otherwise insoluble problems.

The way in which sensory-motor coordination can be exploited also depends on the 
characteristics of the agent. As shown in [2], for example, agents that are able to modify themselves (i.e. to learn) during their interaction with the environment can use sensory-motor coordination to select sensory patterns that are useful to learn (i.e. they can alter the frequency and the order of different sensory patterns to enhance the outcome of the learning process). In this paper however, we will restrict our analysis to the simplest case - that of pure sensory-motor agents which cannot modify themselves during their interaction with the environment and which do not retain any trace of the previously experienced sensory patterns. These agents, by definition, always react in the same way to the same sensory pattern.

\section{How sensory-motor coordination can cope with the perceptual aliasing problem}

One of the most straightforward ways in which sensory and motor processes can be coordinated is to solve the perceptual aliasing problem. Perceptual aliasing, a term coined by Whitehead and Ballard [3], refers to the situation wherein two or more identical sensory patterns require different responses in order to achieve a certain goal. When such a situation occurs (i.e. when an agent receives a sensory pattern that requires different motor responses in different circumstances) the agent should act in order to select other sensory patterns until a sensory patterns which is not affected by the aliasing problem (i.e. an unambiguous sensory pattern) is encountered.

Consider for example the case of a Khepera robot [4] (Fig. 1, left) which is placed in an environment containing two types of object: one with the top painted black, which should be avoided, and one with the top painted white, which should be approached (Fig. 1, right). The Khepera robot is provided with 8 infrared proximity sensors which can detect the bottom part of the obstacles up to a distance of about 3 $\mathrm{cm}$. Moreover, the robot is provided with a K213 linear camera which has 64 photoreceptors and produces a linear image composed of 64 pixels of 256 gray-levels each, subtending a view-angle of $36^{\circ}$. On the motor side, the robot is provided with two wheels controlled by two motors which can rotate in both directions.
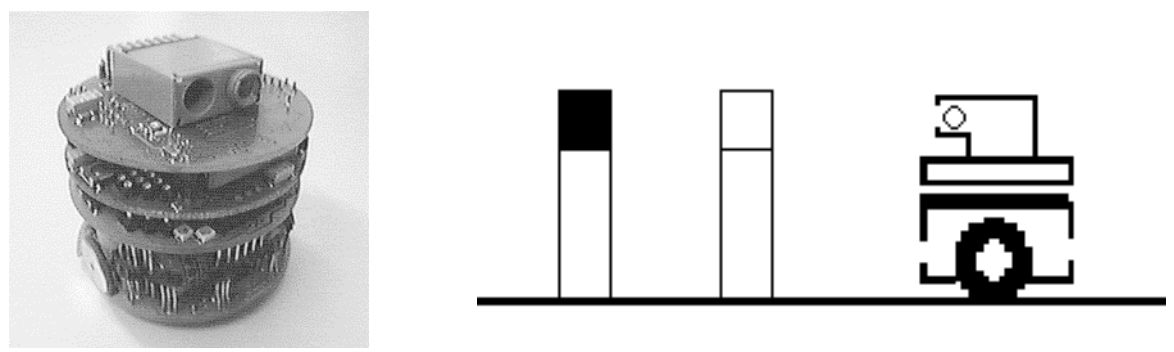

Fig. 1. The robot and the environment. The robot has 8 infrared proximity sensors that can detect the bottom part of the obstacles and a linear camera that can detect the color of the top part of the obstacles.

Every time such a robot approaches an object that does not happen to be in the viewing angle of its camera, it will experience an ambiguous sensory pattern (i.e. a 
sensory pattern which is affected by the aliasing problem). In fact, the same type of sensory pattern will be experienced by the robot both in the case of objects to be approached and in that of objects to be avoided. The obvious solution to this type of problem is to turn toward the object. By turning so as to have the object within the $36^{\circ}$ view angle of the camera the robot will finally receive a unambiguous sensory pattern (i.e. frontal infrared sensors on and white image for objects to be approached and frontal infrared sensors on and a black image for objects to be avoided). The process of selecting sensory patterns that are easy to discriminate through motor actions is usually referred to as active perception [5]. Several examples of processes falling within this category have been identified in natural organisms. As demonstrated by Dill et al. [6], for example, in order to recognize certain visual patterns, drosophila moves so as to shift the perceived image to a certain location in the visual field.

\section{How sensory-motor coordination can simplify hard problems}

In the previous section we saw how the problem of the same sensory pattern requiring different answers can be solved by selecting other unambiguous sensory patterns, if any exist, through sensory-motor coordination. In this section we will investigate problems in which various sensory patterns requiring different motor answers strongly overlap, although not completely. When this happens, agents face hard problems that may be difficult or even impossible to solve. In this section we will try to show how sensory-motor coordination can turn these hard problems into simpler ones.

The distinction between simple and hard problems has been recently formalized by Clark and Thornton [7]. They introduced the term type-2 problems to denote hard tasks in which the problem of mapping input patterns into appropriate output patterns is complicated by the fact that the regularities ${ }^{1}$, which can allow such mapping, are hidden or marginal within the sensory patterns. On the basis of this consideration these authors distinguished type-2 problems (i.e. hard problems) from type-1 problems (i.e. easy problems) in which a sufficient number of regularities are directly available in the sensory patterns.

As claimed by Clark and Thornton, type-2 problems which require complex inputoutput mapping may be reduced to type-1 (tractable) problems by re-coding sensory information so to enhance useful regularities. This can be achieved in two different ways. One possibility is to internally re-code sensory inputs so as to enhance useful regularities. Elman [8], for example, showed how complex tasks which cannot be solved by training a feed-forward neural network using standard back-propagation can be solved if the network is first trained using a simpler subtask and then exposed to the full task. As claimed in [7] and [8] this can be explained by considering that the first learning phase affects how the sensory patterns are re-coded at the level of the internal representations. This re-coding, by enhancing the regularities of the sensory patterns, turns the process of learning the entire task into a type-1 problem [7].

\footnotetext{
1 The term 'regularity' refers to features of the sensory patterns which can be used to discriminate between classes of sensory patterns that require different answers. The exact meaning of the term will become clearer later on.
} 
As shown by Scheier et al. [9], however, agents can also transform a type-2 problem into a type-1 problem by actively structuring their own input through sensory-motor coordination (interestingly, this strategy, as we will see, cannot be used by systems that are passively exposed to sensory states, i.e. systems which are not embodied and situated).

The authors considered the case of a Khepera robot which is supposed to approach large, and to avoid small, cylindrical objects. The robot is provided with six frontal infrared proximity sensors and 2 motor units that encode the actual speed of the two wheels. The environment is an arena surrounded by walls which contains small and large cylinders.

As shown by Nolfi $[10,11]$ who reported a similar experiment in which a Khepera robot had to approach small cylindrical objects and avoid walls, the task of discriminating between the two types of objects is far from trivial for a agent that is required to passively discriminate between the sensory patterns produced by the two objects. This can be explained by considering that the sensory patterns received by the robot depend largely on the distance and on the relative angle between the robot and the objects. As a consequence, the sensory patterns belonging to the two categories largely overlap. "Put differently, the distance in sensor space for data originating from one and the same object can be large, while the distance between two objects from different categories can be small" [9, p. 1559]. On the other hand, as shown by Nolfi $[10,11]$ and by Scheier et al. [9], the task can easily be solved by an agent that is left free to perform sensory-motor coordination. Aside from these similarities the two experiments seem to call for different explanations. For this reason we will describe the experiment reported in [9] first and the experiment described in $[10,11]$ later.

In the simplest experiment reported in [9] Scheier et al. used artificial evolution to select the weights of the robot's neural controllers. Individuals' fitness was increased each time step they were close to a large object and decreased each time step they were close to a small object or a wall. As the authors show, performances increase during the first generations and stabilize in the vicinity of optimal performance after about 40 generations. In other words, while passive systems (i.e. neural networks which are required passively to classify a set of patterns corresponding to two different objects, see $[10,11])$ display poor performance, agents that are allowed to exploit sensory-motor coordination can solve the task easily. The fact that the coordination between the sensory and the motor processes is crucial in solving this task can be clearly demonstrated by observing the behavior of evolved individuals and by observing how the distribution of the sensory patterns changes through the generations.

As reported in [9], the fittest individuals in $86 \%$ of the runs move in the environment until they start to perceive an object (large or small) and then start to circle around the object (the other 14\% stop in front of the objects, although these individuals display significantly poorer performances). At this point the robot continues to circle around large objects while avoiding and abandoning small objects. This circling behavior is crucial to accomplish the discrimination between the two types of object given that the sensory patterns that the robot experiences while circling the small objects are significantly different from those that the robot experiences while circling the large objects. In other words, the sequence of motor actions leading to the circling behavior allows the robot to select sensory patterns that can easily be discerned.

The role of sensory-motor coordination has been further demonstrated by 
measuring the extent to which sensory-patterns belonging to different objects are separated for individuals of different generations (i.e. by measuring the difficulty of the discrimination task for different individuals). Indeed, given that the type of sensory pattern that an individual receives from the environment depends partly on how the individual reacts to each sensory state, individuals who behave differently may face harder or simpler discrimination tasks. To accomplish this measure the authors used the geometric separability index (GSI) proposed by Thornton [12], which can be used to quantify the distinction between type- 1 and type- 2 problems introduced above. In the case of this experiment, the GSI can be used to measure to what extent sensory patterns corresponding to one and the same object are close in sensory space and to what extent sensory patterns corresponding to different objects are separated. GSI is computed by counting the average number of times the sensory pattern nearest to the current sensory pattern falls into the same category. In the case of patterns corresponding to only two categories (small and large objects) this can be calculated in the following way:

$$
G S I(f)=\frac{\sum_{i=1}^{N}\left(f\left(x_{i}\right)+f\left(x_{i}^{\prime}\right)+1\right) \bmod 2}{N}
$$

where $f$ is the category of the object, $x$ are the sensory patterns consisting of $N$ vectors, and $x_{i}{ }^{\prime}$ is the nearest neighbor of $x_{i}$.

As reported in [9], in these experiments the GSI value starts from about 0.5 and monotonically increases during the first 40 generations until it reaches a stable state around 0.9 (note that performance also increases during the first 40 generations). This means that individuals of successive generations increase their ability to coordinate the sensory and motor processes so that experienced sensory patterns corresponding to one object are similar amongst themselves and are different from sensory patterns corresponding to other objects. In other words, evolved individuals are able to transform a type- 2 problem into a type- 1 one.

\section{Exploiting emergent solutions}

In the previous two sections we have considered the case in which it is difficult to react appropriately to part of the sensory patterns because they are affected by the aliasing problem (i.e. the same sensory pattern requires different motor answers) or because the regularities present in the sensory pattern are hidden or marginal (i.e. groups of sensory patterns that require different answers largely overlap). As we showed, both problems can be solved by using sensory-motor coordination. In this section we will examine a similar problem and again we will see how it can be solved by using sensory-motor coordination. The way in which sensory-motor coordination solves this problem, however, seems to be qualitatively different from the two cases described above. Indeed, as we will see, this way of solving the problem is effective also in cases in which all sensory patterns are affected by the aliasing problem or, in other words, cases in which the sensory data do not contain any regularities at all.

As mentioned above, Nolfi conducted an experiment in which a Khepera robot was required to discriminate between walls and cylindrical objects by finding and remaining close to the latter $[10,11]$. The environment was an arena of $60 \times 35 \mathrm{~cm}$ 
surrounded by walls with a cylindrical object with a diameter of about $3 \mathrm{~cm}$ randomly distributed inside it. To develop the control system for this robot Nolfi used artificial evolution. Individuals' fitness was increased each time step they were close to the cylindrical object. After a few generations the best individuals succeeded in ending up close to the cylindrical object within 500 cycles most of the times. This means that they were able to discriminate between the two objects, avoiding walls and remaining close to the cylinders (see Fig. 2, thick line).

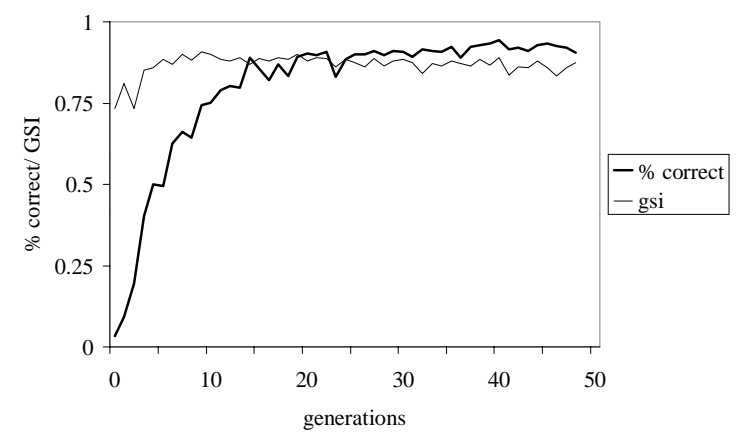

Fig. 2. Thick line: Percentage of times the best individuals of each generation succeed in ending up close to the cylindrical object after 500 cycles. Thin line: GSI of the sensory patterns experienced by individuals of successive generations. Average results of 10 replications.

Evolved individuals do not circle around objects (as in the case of the experiment described in the previous section). On the other hand, all evolved individuals start to move back and forth and/or left and right when they approach the cylinder. This emergent behavior can be described as a dynamical system and the relative positions with respect to an object in which individuals start to move back and forth or left and right while remaining in proximity to the object can be described as an attractor since the robot's trajectory converges on the same relative positions regardless of the direction of approach to the target. This can be seen in Fig. 3 which shows the trajectory of the movements produced by an evolved individual while approaching walls or cylinders (top and bottom, respectively). As can be seen, when the individual reaches a distance of about $20 \mathrm{~mm}$ from an object it avoids walls while it continues to approach targets until it reaches the attractor area located at a distance of about 15 $\mathrm{mm}$ and an angle of about 45 degrees. The trajectory of the motor responses in this area all converge toward the center of the area itself allowing the individual to keep more or less the same relative position with respect to the cylinder.

At this point we may wonder about the role of sensory-motor coordination in this type of emergent behavior. Can we conclude that, as in the experiments described in the previous section, sensory-motor coordination allows individuals to be exposed to data in which groups of sensory patterns belonging to different objects (walls and cylinders in this case) do not strongly overlap?

One way to answer this question is to look at the GSI index through generations. As we can see from Fig. 2 it stabilizes around 0.8 after the very first generation, while performance continues to increase through 50 generations. The fact that performance continues to increase significantly while GSI stabilizes and the fact that it stabilizes at a lower value with respect to the experiments described in the previous section (about 0.8 instead of 0.9 ) suggests that in this case the ability to experience sensory patterns 
belonging to two objects (walls and cylinders in this case) that are easy to discriminate plays a less important role.
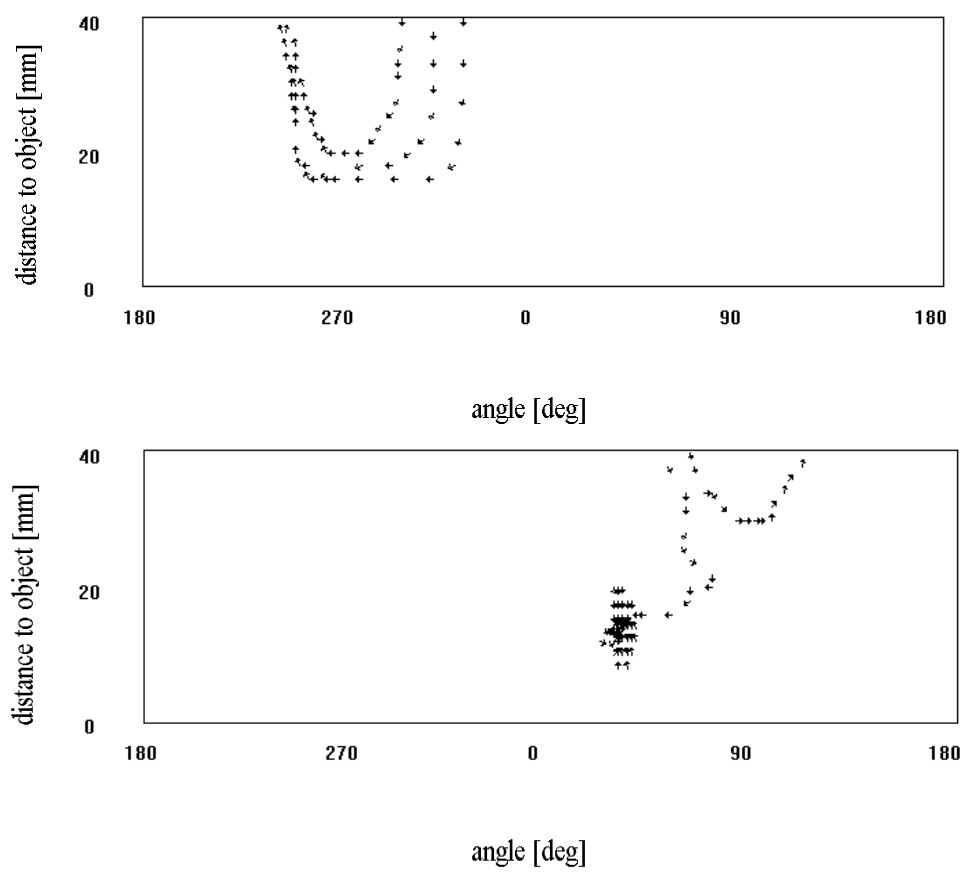

Fig. 3. Angular trajectories of an evolved individual close to a wall (top graph) and to a target (bottom graph). The picture was obtained by placing the individual in a random position in the environment, leaving it free to interact with the environment for 500 cycles, and recording the change in the relative positions with respect to the two objects for distances lower than $40 \mathrm{~mm}$. Angle 0 corresponds to objects in front of the robot. For the sake of clarity arrows are used to indicate the relative direction but not the amplitude of the movements.

Another way to investigate the role of sensory-motor coordination in this experiment is to study another case in which the environmental conditions are designed to prevent the robot from behaving in such a way that the separability between groups of sensory patterns belonging to different objects increases. Consider the case of a simulated agent which lives in a circular strip divided into 40 cells (20 cells on the left and 20 on the right). At each time step the agent occupies one single cell and perceives the sensory state associated with the cell. There are 20 different sensory states that the agent can perceive, numbered from 0 to 19 , which are each associated with a single cell both in the left and in the right part of the environment in a randomly different order (see Fig. 4). The agent can react to the current sensory state in two different ways (move one cell clockwise or anti-clockwise) and has the goal of reaching and remaining in the left part of the environment.

The agents have a neural network with 20 input units which locally encodes the corresponding perceived sensory state and 1 output unit which binarily encodes one of the two possible actions. As a consequence only one sensory unit is activated each time step. Weights can assume only two values ( 0 or 1$)$. As a consequence, the weight 
connecting the input unit corresponding to the current sensory state to the output unit determines the motor reaction of the agent at each time step (move clockwise or anticlockwise if the weight is 0 or 1 , respectively). Individuals do not have any memory of the previously experienced sensory states (i.e. they always react in the same way to a given sensory state).
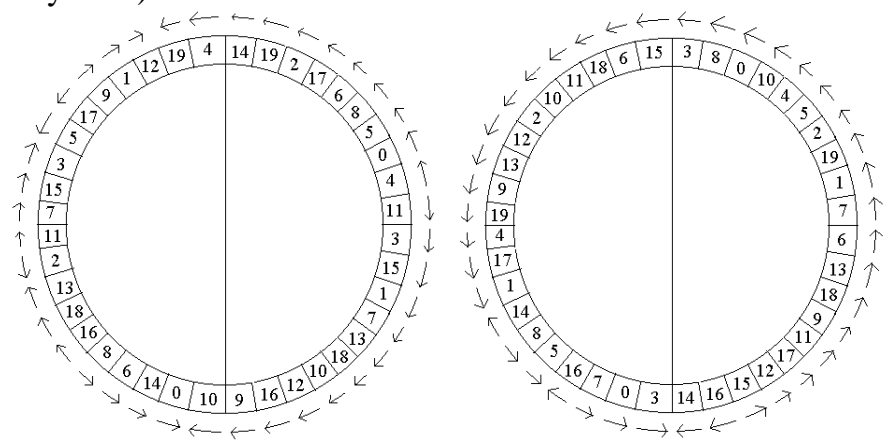

Fig. 4. Two environments. The numbers represent the sensory state experienced by the agent in each cell. Each different sensory state is present once in the left and once in the right part of the environment. Arrows indicate the motor reaction of a typical evolved agent.

What is interesting about this experimental situation is that all possible sensory states are affected by the aliasing problem. For each possible sensory state experienced, in fact, the animat has a $50 \%$ probability of being in the left or in the right part of the environment. In other words, in this case it is impossible to use sensory-motor coordination to select sensory states which are not affected by the aliasing problem or sensory states in which groups belonging to different categories (left and right side of the environment in this case) do not strongly overlap (in this case, in fact, all 20 different sensory states are equidistant in the input space).

Despite this, if we evolve a population of agents by selecting those that ended up in the left part of the environment after 200 cycles, after a few generations we obtain individuals that are able to move away from the right part of the environment and to remain in the left part ${ }^{2}$. The way in which evolved individuals solve this problem can be seen by observing the arrows in Fig. 4. In the right part of the environment individuals consistently move clockwise or anti-clockwise until they abandon the right side. Conversely, in some areas of the left side of the environment, individuals start to move back and forth, although remaining there for the rest of their life. Note that the way an individual reacts to a particular sensory state (for example the fact that the individual shown on the left side of Fig. 4 reacts clockwise to the sensory state ' 3 ') does not have any function in itself. The way in which an evolved individual reacts to a certain sensory state makes sense only if we consider how it reacts to all other sensory states. Agents solve the problem by reacting anticlockwise to the upper cells and clockwise to the lower cells in the right portion of the environment. This implies

2 Evolving individuals were allowed to "live" for 100 epochs, each epoch consisting of 200 actions. Connection weights were binarily represented in the genotype which was 20 bits long. Population size was 100 . The best 20 individuals of each generation were allowed to reproduce by generating 5 copies of their genotype with $2 \%$ of their bits replaced with a new randomly selected value. The experiment was replicated 10 times. 
that there is only a single point on the right side of the environment, no matter where, in which two adjacent cells are responded to in different ways. This guarantees that the agent quickly leaves the undesired right side and moves to the desired left side. When the agent finds itself on the left side of the environment, the different spatial distribution of the cells on the left side with respect to the right side with their associated sensory states will ensure, with a very high probability, that the agent moves within the left side of the environment without ever leaving it. Therefore the problem is solved by implicitly exploiting the particular spatial distribution of sensory patterns in the environment.

Note that the way in which these simulated agents solve their task closely resembles the strategy adopted by the evolved robots described earlier in this section. The environment has only one dimension in this experiment while it has two dimensions in the previous experiment (the third dimension is irrelevant given that the robot can only move in two dimensions). This explains why agents move clockwise or anticlockwise in these experiments while robots moved back and forth and left and right in the previous experiments. On the other hand, the type of strategy adopted by evolved individuals is the same: react to sensory states to produce attractors (i.e. set of motor actions that result in a set of movements that, summed together, allow the individual to remain in the same position) in the left part but not in the right part of the environment in this experiment; react to sensory states to produce an attractor close to cylindrical objects but not close to walls in the previous experiment.

Obviously this task cannot be solved without exploiting sensory-motor coordination (e.g. it is impossible to train a network to produce different outputs for sensory patterns belonging to the left and to the right part of the environment). On the other hand, it is not possible either to solve this task: (1) by using sensory-motor coordination to select sensory states not affected by the aliasing problems or (2) by selecting sensory states in which groups belonging to different categories do not strongly overlap. This means that there is a third way in which sensory-motor coordination can solve hard tasks. This third way relies on emergent solutions (i.e. simple solutions relying on the dynamical interaction between the agent and the environment).

\section{Conclusions}

We have seen how sensory-motor coordination (i.e. the ability to select sensory patterns that are useful for some purpose through motor actions) can solve hard problems in three different ways: (1) by selecting sensory patterns which are not affected by the aliasing problem and avoiding those that are; (2) by selecting sensory patterns in which groups of patterns requiring different motor responses do not strongly overlap; (3) by reacting to each sensory pattern in a way that maximizes performance in view of how the agent reacts to all other sensory patterns.

The third way, which we have called emergent behavior, is in a way more radical than the other two categories. When we claim that a sensory pattern is affected by the aliasing problem or that two groups of sensory patterns that require different responses overlap strongly we are implicitly assuming a certain behavioral solution to a given task (e.g. we are assuming that the agent should react differently to patterns belonging to different objects or to different sides of the environment). However, 
often tasks can be solved in different ways and only some of these ways may present problems such as aliasing or lack of regularities among groups of sensory patterns that require different answers. Let us consider the last experiment. When we first think about this task we assume that the only way to solve it is to react differently to sensory states present in the left and in the right part of the environment (at least to some of them). When we then realize that all sensory patterns are present on both sides and equally distant in the input space we feel that there is no way to solve the problem (at least without taking into account previously experienced states). However, when we observe the behavior of the evolved agent, we see that there is a completely different way to solve the problem which does not require reacting differently to sensory states lying on the two sides.

When we leave individuals free to find their own solution to a task by interacting with the external environments, two different processes take place. On one hand, individuals select those strategies that are less affected by the aliasing problem or by the lack of regularities within groups of sensory patterns requiring different answers. On the other hand, given a certain selected strategy, individuals try to use sensorymotor coordination to avoid sensory patterns affected by aliasing problems and to experience sensory patterns so to increase regularities within groups of sensory patterns requiring different answers.

\section{References}

1. Pfeifer, R. \& Scheier, C. Sensory-motor coordination: The metaphor and beyond. Robotics and Autonomous Systems. 20 (1997) 157-178.

2. Nolfi, S. Parisi, D.: Learning to adapt to changing environments in evolving neural networks. Adaptive Behavior. 1: (1997) 99-105

3. Whitehead, S.D. \& Ballard, D. H.: Learning to perceive and act by trial and error. Machine Learning. 7 (1991) 45-83.

4. Mondada, R., Franzi, E. \& Ienne, P.: Mobile robot miniaturization: A tool for investigation in control algorithms: In: T. Yoshikawa \& F. Miyazaki (eds.): Proceedings of the Third International Symposium on Experimental Robots, Kyoto, Japan (1993)

5. Bajcsy, R.: Active Perception. Proceedings of the IEEE (76) 8: (1988) 996-1005

6. Dill, M., Wolf, R., Heisenberg, M.: Visual pattern recognition in drosophila involves retinotopic matching. Nature. 355: (1993) 751-753.

7. Clark, A. \& Thornton, C.: Trading spaces: Computation, representation, and the limits of uniformed learning. Behavioral and Brain Sciences. 20 (1997) 57-90.

8. Elman, J.L.: Learning and development in neural networks: The importance of starting small. Cognition. 48 (1993) 71-99.

9. Scheier, C., Pfeifer, R. \& Kunyioshi, Y. Embedded neural networks: exploiting constraints. Neural Networks. 11: (1998) 1551-1596.

10.Nolfi, S.: Adaptation as a more powerful tool than decomposition and integration. In: T. Fogarty and G. Venturini (Eds), Proceedings of the workshop on Evolutionary computing and Machine Learning, 13th International Conference on Machine Learning, Bari (1996)

11.Nolfi, S.: Evolving non-trivial behavior on autonomous robots: Adaptation is more powerful than decomposition and integration. In T. Gomi (ed.): Evolutionary Robotics, Kanata, Canada: AAI Books (1997)

12.Thornton C.: Separability is a learner's best friend. In: J.A. Bullinaria, D.W. Glasspool, \& G. Houghton (eds.): Proceedings of the Neural Computation and Psychology Workshop: Connectionist Representations, London, Springer (1997). 